\title{
Author Correction: The delay of shock breakout due to circumstellar material evident in most type II supernovae
}

F. Förster (D), T. J. Moriya (D), J. C. Maureira, J. P. Anderson (D), S. Blinnikov (D), F. Bufano, G. Cabrera-Vives, A. Clocchiatti, T. de Jaeger, P. A. Estévez, L. Galbany DD, S. González-Gaitán, G. Gräfener, M. Hamuy, E. Y. Hsiao, P. Huentelemu, P. Huijse (D), H. Kuncarayakti DD, J. Martínez DI, G. Medina, F. Olivares E. ID, G. Pignata, A. Razza, I. Reyes, J. San Martín, R. C. Smith, E. Vera, A. K. Vivas, A. de Ugarte Postigo (D), S.-C. Yoon, C. Ashall, M. Fraser, A. Gal-Yam (D), E. Kankare (D), L. Le Guillou, P. A. Mazzali, N. A. Walton and D. R. Young (D)

Correction to: Nature Astronomy https://doi.org/10.1038/s41550-018-0563-4, published online 3 September 2018.

In the version of this Article originally published, the authors Pablo Huijse and Pablo Huentelemu were mistakenly affiliated with the University of California, Berkeley, and their affiliation to the University of Chile was omitted. Pablo Huijse's affiliation to the Universidad Austral de Chile was also omitted.

Pablo Huijse's affiliation has now been corrected to: Millennium Institute of Astrophysics, Santiago, Chile; Department of Electrical Engineering, University of Chile, Santiago, Chile; Instituto de Informática, Universidad Austral de Chile, Valdivia, Chile.

Pablo Huentelemu's affiliation has now been corrected to: Department of Electrical Engineering, University of Chile, Santiago, Chile. 